\title{
3D Geological Pressure Prediction for a Block of the Jequitinhonha Basin
}

Fernando T. B. de Andrade, Lourenildo W. B. Leite, Wildney W. S. Viera, UFPA, Brazil

Copyright 2019, SBGf - Sociedade Brasileira de Geofísica.

This paper was prepared for presentation at the $16^{\text {th }}$ International Congress of the Brazilian Geophysical Society, held in Rio de Janeiro, Brazil, August 19-22, 2019.

Contents of this paper were reviewed by the Technical Committee of the $16^{\text {th }}$ International Congress of The Brazilian Geophysical Society and do not necessarily represent any position of the SBGf, its officers or members. Electronic reproduction or storage of any part of this paper for commercial purposes without the written consent of The Brazilian Geophysical Society is prohibited.

\section{Abstract}

The prediction of pressure distribution in the subsurface is relevant for mapping and extending the potential zones of a productive reservoir, that is important in prospecting for hydrocarbons, oil and gas. The objective of this work was to structure a seismic-stratigraphical framework, to apply a sequence of calculus for modeling and imaging part of the Jequitinhonha basin (marine, east of the State of Bahia) to evaluate the pressure distribution in the subsurface, that can be used to evaluate the potential for producing oil and gas. In addition, the main interest is to map the reservoir, as well as its lateral and vertical extensions. The knowledge required for the modeling are the distributions of velocities $V_{P}$ and $V_{S}$, and density $\rho$, of the block under investigation, which were obtained empirically from seismic-stratigraphic interpretations of time-migrated data with the CRS technology. These distributions can also be obtained in an independent step of velocity analysis based on seismic sections. Petrophysical information and empirical models that provide a relationship between velocities, density and porosity can also be incorporated. The model admits the vertical gravity load as pressure agent on the geological formations and does not take into account the effects of curvatures, faulting, diagenesis, and lateral tectonic events. We present a robust pressure prediction for a 3D interpolated model, where we map the extension of the low pressure zones (possible reservoir) for a significant geological practical application.

\section{INTRODUCTION}

This work had for motivation the geomechanical problem related to productive sedimentary basins of oil and gas, or basins with potential for exploration. Thus, we are dealing here specifically with the prediction of pressure in the Jequitinhonha basin, where six seismic lines were used, and separated into two groups: three lines in the NESW (L214-0266, L214-0268, L214-0270) and three in the NW-SE direction (L214-0297, L214-0302 e L214-0303). Figure 1 shows the location and arrangement of seismic lines used.

The methodology was composed by a sequence of processing techniques as CRS stack, CRS migration, interpretation of reflectors, time-depth conversion and
Figure 1: Location of the six seismic lines used in the Jequitinhonha basin.

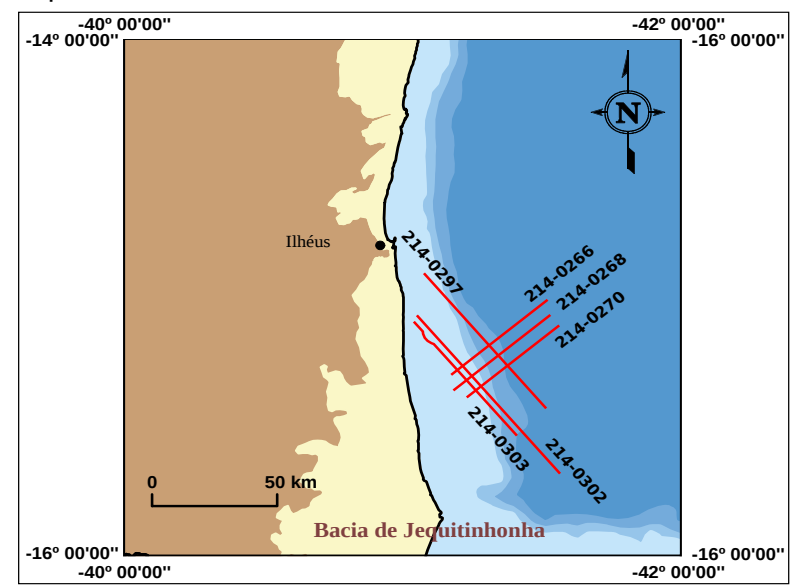

construction of a controlled empirical model for $\mathrm{P}$ and $\mathrm{S}$ wave velocities and density, necessary for the prediction of subsurface pressure. The final aim is to map zones of low and high pressure, that act as natural pumps for the accumulation of fluids (Payton, 1977; Mann, 2002; Cohen and Stockwell, 2005).

The seismic interpretation serves to recognize textures existing between the main patterns of the reflectivity field, where the basic principles considered are: resolution (vertical), continuity (horizontal), texture (of the intervals between dominant events), and frequency content (low, medium, high).

The Regência petroleum system is responsible for all hydrocarbon occurrences in the Jequitinhonha Basin. However, it is necessary to emphasize that a rock is a reservoir candidate if it fulfills the mechanical-static conditions of pressure (Sibiryakov et al., 2014; Andrade, 2018).

Figures 2 and 3 show the interpreted sections using the CRS time migration, which were used to construct the different cubes of information. The interpretation was based on the primary reflection field, that showed good lateral continuity and systematic texture of the macro segments, where the sequences and structures are indicated by letters and numbers. The blue lines mark the main reflectors, the black lines mark the faults and the red lines mark the main multiples.

\section{PRESSURE PREDICTION}

The main physical parameter governing the pressure prediction phenomenon is the measure of discontinuity represented by the ratio $\gamma=V_{S} / V_{P}$ across the geologic 
Figure 2: Seismic interpretations. Lines in NE-SW direction.
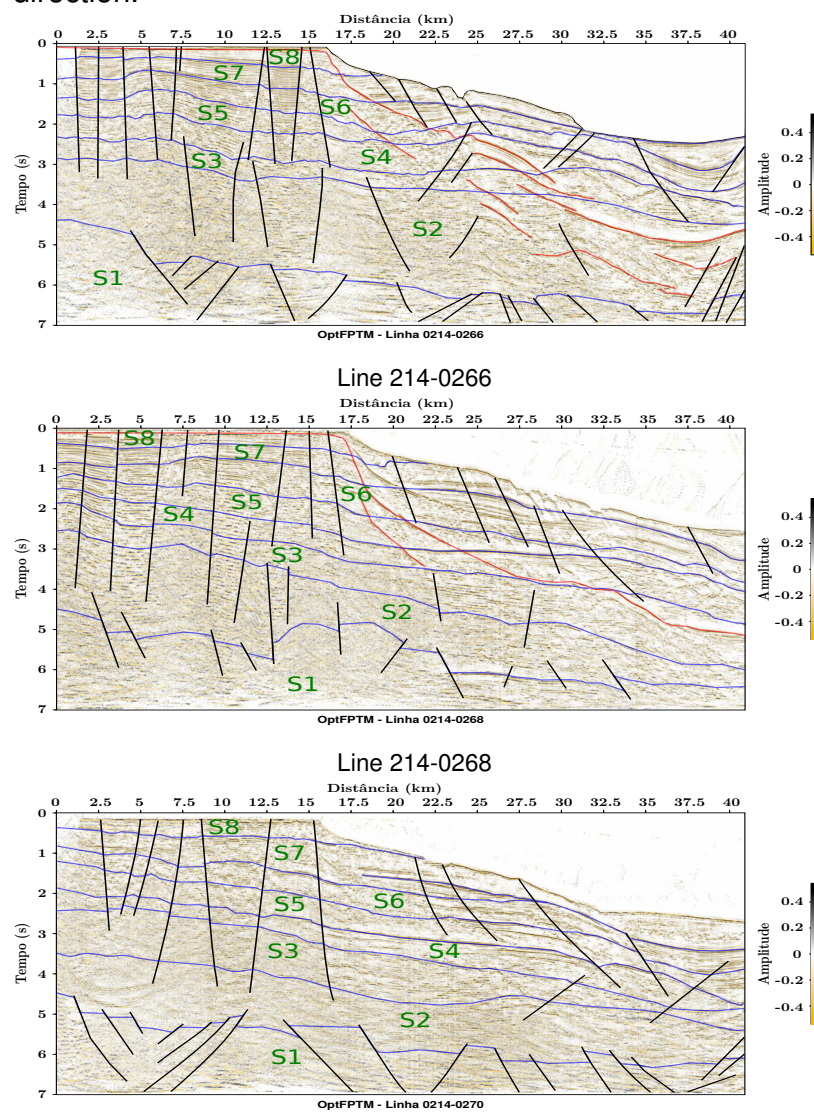

Line 214-0270

interfaces. As a result of this investigation, Sibiryakov et al. (2015) is an example that deals with different aspects of this research topic.

A result related to behavior of the gamma ratio $(\gamma)$, is that an anticline is not necessarily the only structural condition for potential zone to be a oil and gas accumulator. A trap can be present in a horizontal structure if the discontinuity in the Gamma ratio is positive, from top to bottom, around an interface. This physical condition is responsible for producing a pressure discontinuity, so that there will be a sufficiently lower pressure zone under the interface than above it, generating a fluid attraction surface.

The continuous linear elastic stress fields, $\sigma=\sigma(x, y, z)$, and deformation, $\varepsilon=\varepsilon(x, y, z)$, are related by Hooke's law, described as tensors, functions of space, which are represented by nine components. For the general anisotropic media, the tensors $(\sigma)$ and $(\varepsilon)$ obey the spatial coordinate rotation by the relation: $\sigma_{i j}=\sum_{k, l} a_{i j k l} \sigma_{k l}^{\prime}$, and $\varepsilon_{i j}=\sum_{k, l} b_{i j k l} \varepsilon_{k l}^{\prime}$, where the coefficients $a_{i j k l}$ and $b_{i j k l}$ define a new plane with respect to a reference system.

The elastic linear relationship between stress and strain is given by the generalized Hooke's law: $\sigma_{i j}=\sum_{k, l} c_{i j k l} \varepsilon_{k l}$. In this description, the first index $(i)$ in $\sigma_{i j}$ and in $\varepsilon_{i j}$ represents the plane direction, and the second $(j)$ the component direction.

The stress state is represented at a point $Q$ by a matrix $\mathbf{S}$
Figure 3: Seismic interpretations. Lines in NW-SE direction.
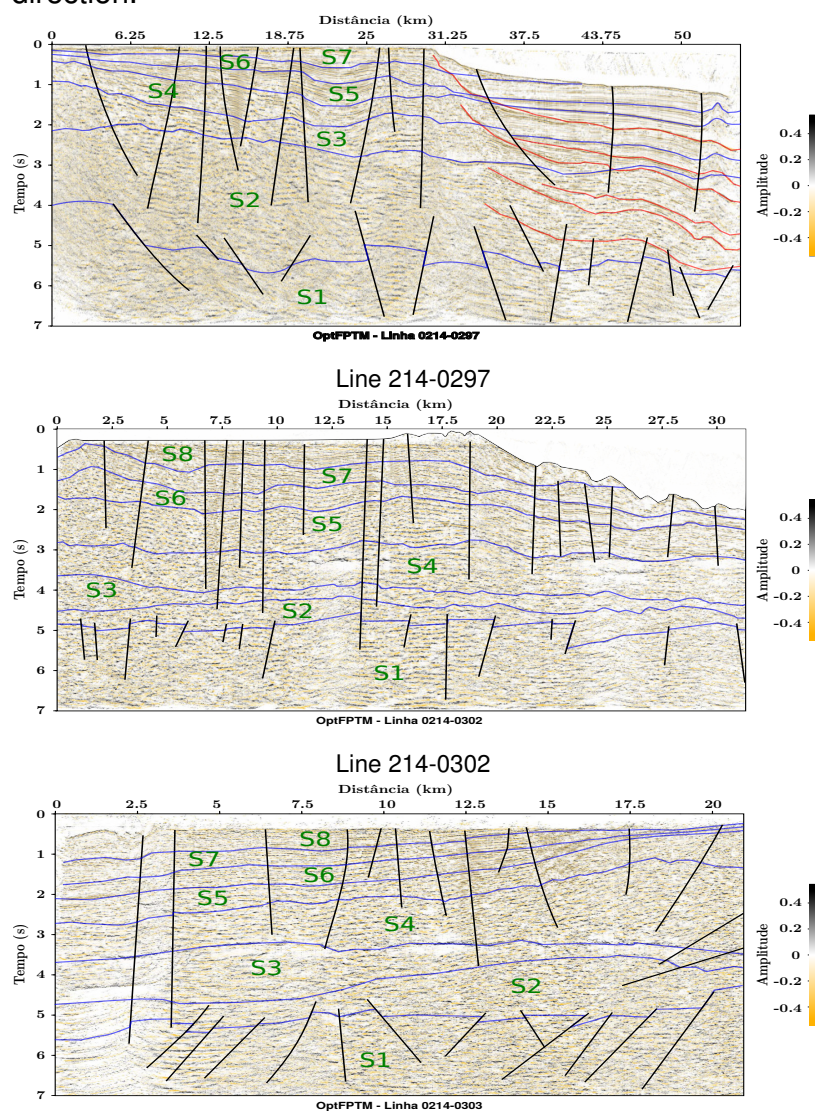

Line 214-0303

with elements $\sigma_{i j}$.

\section{Stress states}

The stress states matrix can be decomposed into three parts in the form: $\mathbf{S}=\mathbf{S}_{\mathbf{0}}+\mathbf{S}_{\mathbf{D}}+\mathbf{S}_{\mathbf{N}}$, which allows a physical interpretation according to Persen (1975).

For the state $\mathbf{S}_{\mathbf{0}}$ we simply have $\mathbf{S}_{\mathbf{0}}=\left\{P_{H} \delta_{i j}\right\}$ where $P_{H}=$ $\frac{1}{3}\left(\sigma_{x x}+\sigma_{y y}+\sigma_{z z}\right)$ is the sum of the normal stresses which define the so-called hydrostatic pressure, and this state is present in any plane around a point $Q$.

For the state $\mathbf{S}_{\mathbf{D}}$ one has that:

$$
\mathbf{S}_{\mathbf{D}}=\left[\begin{array}{ccc}
\sigma_{x x}-P_{H} & \frac{1}{2}\left(\sigma_{x y}+\sigma_{y x}\right) & \frac{1}{2}\left(\sigma_{x z}+\sigma_{z x}\right) \\
\frac{1}{2}\left(\sigma_{x y}+\sigma_{y x}\right) & \sigma_{y y}-P_{H} & \frac{1}{2}\left(\sigma_{y z}+\sigma_{z y}\right) \\
\frac{1}{2}\left(\sigma_{x z}+\sigma_{z x}\right) & \frac{1}{2}\left(\sigma_{z y}+\sigma_{y z}\right) & \sigma_{z z}-P_{H}
\end{array}\right] .
$$

Applying the symmetry property to the above matrix (1): $\sigma_{x y}=\sigma_{y x}, \sigma_{x z}=\sigma_{z x}, \sigma_{y z}=\sigma_{z y}, \mathbf{S}_{\mathbf{D}}$, results in a state represented by:

$$
\mathbf{S}_{\mathbf{D}}=\left[\begin{array}{ccc}
\sigma_{x x}-P_{H} & \sigma_{x y} & \sigma_{x z} \\
\sigma_{x y} & \sigma_{y y}-P_{H} & \sigma_{y z} \\
\sigma_{x z} & \sigma_{y z} & \sigma_{z z}-P_{H}
\end{array}\right]
$$

what places the deviatoric state along the diagonal (normal stresses), where the hydrostatic state is subtracted, remaining the nonhydrostatic state. The state $\mathbf{S}_{\mathbf{D}}$ is 
generally called deviatoric, because it represents the degree to which a certain state of tension deviates from the hydrostatic state.

Finally, for the state $\mathbf{S}_{\mathbf{N}}$, we have:

$$
\mathbf{S}_{\mathbf{N}}=\left[\begin{array}{ccc}
0 & \frac{1}{2}\left(\sigma_{x y}-\sigma_{y x}\right) & \frac{1}{2}\left(\sigma_{x z}-\sigma_{z x}\right) \\
\frac{1}{2}\left(\sigma_{x y}-\sigma_{y x}\right) & 0 & \frac{1}{2}\left(\sigma_{y z}-\sigma_{z y}\right) \\
\frac{1}{2}\left(\sigma_{x z}-\sigma_{z x}\right) & \frac{1}{2}\left(\sigma_{z y}-\sigma_{y z}\right) & 0
\end{array}\right] .
$$

Similarly, by applying the symmetry property, the state $\mathbf{S}_{\mathbf{N}}$ is simplified to the null state, that is, $\mathbf{S}_{\mathbf{N}}=[\mathbf{0}]$.

For an isotropic, linear elastic, medium, the relation between stress and strain is represented by Hooke's law: $\sigma_{i j}=\lambda \theta \delta_{i j}+2 \mu \varepsilon_{i j}$, where $\lambda$ and $\mu$ are the Lamé elastic parameters, and $\delta_{i j}$ the Kronecker delta $\left(\delta_{i j}=0\right.$, if $i \neq j$ and $\delta_{i j}=1$, if $i=j$ ).

The nondimensional parameter $\theta$ represents the cubic dilation, and is given by the divergence of the displacement vector $\vec{u}$ as: $\theta=\nabla \cdot \vec{u}=\frac{\partial u_{x}}{\partial x}+\frac{\partial u_{y}}{\partial y}+\frac{\partial u_{z}}{\partial z}$.

The nondimensional tensor components $\varepsilon_{i j}$ are defined in terms the displacement components $u_{i}$ in the form: $\varepsilon_{i j}=\frac{1}{2}\left(\frac{\partial u_{i}}{\partial x_{j}}+\frac{\partial u_{j}}{\partial x_{i}}\right)$. Besides that, the linear shearextensional tensor, an nondimensional rotation tensor is also expressed as: $\varphi_{i j}=\frac{1}{2}\left(\frac{\partial u_{i}}{\partial x_{j}}-\frac{\partial u_{j}}{\partial x_{i}}\right)$.

\section{Physics of geological solid medium}

Since this phenomenon is also related to elastic wave propagation, the system equations of motion are resumed to the form: $\frac{\partial \sigma_{i j}}{\partial x_{j}}=\rho \frac{\partial^{2} u_{i}}{\partial t^{2}}, \quad(i, j=1,2,3), \quad$ or $\quad(i, j=x, y, z)$. This means that the spatial stress variation is related to the inertial forces (per unit volume), not considering internal forces in this case.

The basic seismic velocities ( $P$ and $S$ ) for elastic, homogeneous, isotropic media are given by: $V_{\mathrm{P}}=$ $\sqrt{(\lambda+2 \mu) / \rho}, V_{\mathrm{S}}=\sqrt{\mu / \rho}$, where $\mu$ is the shear module, $\rho$ is the volumetric density, and $\lambda$ is related to the Bulk and shear modules. From the above relations, the shear module is calculated by: $\mu=V_{\mathrm{S}}^{2} \rho, \lambda=V_{\mathrm{P}}^{2} \rho-2 \mu=\left(V_{\mathrm{P}}^{2}-\right.$ $\left.2 V_{\mathrm{S}}^{2}\right) \rho$, and the ratio $\gamma=V_{\mathrm{S}} / V_{\mathrm{P}}$.

Density is usually admitted as a parameter that varies slowly with depth, from the earth's surface to the top of the target interface. Nevertheless, in some geological situations the density discontinuity may be relatively high. In the present case, the density is modeled in 2D, $\rho=$ $\rho(x, z)$, and is integrated in the calculation grid. The empirical relationship $\rho \approx 1.741 V_{S}^{0.25}$ proposed by Gardner et al. (1974) was used, because it represents an average on different types of rock.

The system of differential equations to be integrated, that corresponds to the description of the static problem, has the null time variation, governing a part (layer) of the medium, which is summarized to the form: $\frac{\partial \sigma_{l j}}{\partial x_{j}}=\rho g \delta_{3 j}$. This means that the sum for the horizontal stress variations are zero, and for the vertical component is controlled by the gravitational load of the subsurface expressed in terms of force per unit area $(\rho g)$. Therefore, the lateral tectonic stresses are not taken into account in this model. Also, in other cases the quantities $\rho$ and $g$ can be considered as spatial functions; that is, $\rho=\rho(x, y, z)$ and $g=g(x, y, z)$.
We describe as a first model a medium formed by planehorizontal layers. The equilibrium equation for the linear elastic medium for each layer is given by: $\frac{\partial \sigma_{i k}}{\partial x_{k}}=\rho g_{i}$, where $\sigma_{i k}$ represents the components of the stress tensor, $\rho$ the rock density, and $g_{i}$ the gravity acceleration. For the case of vertical gravity, $g_{i=z}(z) \approx g$, admitted as constant for a small depth variation, is simplified to the form: $\frac{\partial \sigma_{z z}}{\partial x_{z}}=\rho g$. This equation has an elementary solution that is given by: $\left.\sigma_{z z}\right|_{z=z_{0}}=\int_{z=0}^{z=z_{0}} \rho g d z=\rho g z_{0}=P_{0}\left(z_{0}\right)$, where $P_{0}=\rho g z_{0}$ is the overload rock weight per unit area, i.e., the vertical pressure due to the overload at any depth $z_{0}$.

For the present model, the vertical stress, $\sigma_{z z}(z)$, is defined as equal to overloading layer, i. e., $\sigma_{z z}=P_{z}=P_{0}(z)$. The horizontal stress, $\sigma_{x x}(z)$, considering that $\sigma_{y y}=\sigma_{x x}$, is smaller than vertical stress, $\sigma_{z z}$, and is demonstrated to be given by $\sigma_{x x}=P_{x}=P_{0}\left(1-2 \gamma^{2}\right)$, where $P_{0}=P_{0}(z)$, $\gamma=\gamma(z)=V_{\mathrm{S}}(z) / V_{\mathrm{P}}(z)$.

The scalar invariant hydrostatic pressure field, $P(z)=P_{H}$, was defined above as the average $P=P_{H}=\frac{1}{3}\left(\sigma_{x x}+\sigma_{y y}+\right.$ $\left.\sigma_{z z}\right)$. Using Hooke's generalized law, it is demonstrated to be given by $P=P_{H}=\left(\lambda+\frac{2}{3} \mu\right) \theta$, where $\theta(z)$ is the cubic dilation, and $\lambda(z)$ and $\mu(z)$ are Lamé's parameters described before.

Another important physical feature is the pressure discontinuity at the interfaces between layers $\left(\Delta P=P^{+}\right.$ $P^{-}$), at the depth $z$ (positive down), which exists if the velocity ratio $\gamma$ exhibits a discontinuity. Considering the case of a medium formed by plane-horizontal layers, and Hooke's law, it is shown that the discontinuity $\Delta P$ is given by $\Delta P(z)=\frac{4}{3}\left(\gamma_{1}^{2}-\gamma_{2}^{2}\right) P_{0}(z)$, where $\gamma_{1}$ is the upper layer and $\gamma_{2}$ the lower layer parameter, across the interface positioned at the depth $z$, and the overload pressure varies by positive and negative jumps. This idea may seem a little strange in simple geological descriptions, but it is a fact related to the non-elementary behavior of stress in solids.

The intensity of tangential stress is a way of measuring the mechanical instability responsible for the ability to destroy the granular structure and produce fracture of the solid rock, and the following result is obtained: $P_{T}=\frac{1}{2}\left(\sigma_{z z}-\right.$ $\left.\sigma_{x x}\right)=\gamma^{2} P_{z}$, and again the above result also depends on the $\gamma$ ratio.

\section{RESULTS}

The velocity and Gamma ratio distributions used are based on controlled empirical models presented in the table (1).

Table 1: Parameter values used in the empirical models.

\begin{tabular}{c|c|c|c|c|c}
\hline Value & S1 & S2 & S3 & S4 & S5 \\
\hline$\gamma_{0}$ & 0 & 0.677 & 0.677 & 0.677 & 0.677 \\
$V_{P 0}$ & 1500 & 1900 & 2200 & 2350 & 2500 \\
$k_{P}$ & 0 & 0.01 & 0.01 & 0.01 & 0.05 \\
$k_{g}$ & 0 & 0.0001 & 0.0001 & 0.0001 & 0.0001 \\
\hline \multicolumn{2}{c|}{ Value } & $\mathbf{S 6}$ & $\mathbf{S 7}$ & $\mathbf{S 8}$ & $\mathbf{S 9}$ \\
\hline \multicolumn{2}{c|}{$\gamma_{0}$} & 0.687 & 0.807 & 0.677 & 0.570 \\
$V_{P 0}$ & 3000 & 3440 & 4200 & 5200 \\
$k_{P}$ & 0.02 & 0.025 & 0.075 & 0.2 \\
$k_{g}$ & 0.0001 & 0.0001 & 0.0001 & 0.0001 \\
\hline
\end{tabular}

For lack of space, the next eight figures exhibits only 
the resulting maps for line L214-0266, due to the good resolution presented by this data. The results for all lines are presented by Andrade (2018).

Figure (4) shows the P velocity empirical model used, where we have the main layers represented in the following form: S1 corresponds to the water layer (dark blue); S6 corresponds to a layer above the reservoir (light blue); S7 corresponds to the layer that includes the reservoir (light green); S8 corresponds to the layer that includes the source rock (yellow); and S9 corresponds to the basement (dark red).

The formula for the velocity $V_{P}$ is given by $V_{P}(z)=V_{P 0}+$ $k_{P} z$, for the Gamma ratio $\gamma(z)=\gamma_{0}+k_{g} z$, and for the density $\rho(z) \approx 1.741 V_{S}(z)^{0.25}$. These formulas were used to construct the parameter distributions with jumps at the interfaces, and smooth vertical gradient in each layer. To this information we used the results based on the observations by Gregory (1976) for the values of $\gamma$ based on the Poisson's ratio.

The basic sections for the pressure prediction are the velocity $V_{P}$ and the ratio $\gamma$ (figures 4 and 5), from where $V_{S}$ (figure 6) and all others sections are calculated. In order to obtain these two first sections, we started from the CRS stacked interpreted sections, where the source rock and reservoir formations were selected based on geological information.

Figure 7 shows the density distribution, $\rho$, of the example, which also followed the mixed pattern similar to those for $V_{P}$, that is, with jumps and smooth vertical gradient.

Figure 8 shows the vertical pressure, $P_{z}=\sigma_{z z}$, shows a very smooth behavior as expected, and represents the overload pressure field. On the other hand, figure 9 shows the horizontal pressure, $P_{x}=\sigma_{x x}=P_{0}\left(1-2 \gamma^{2}\right)$, with a very differentiated and special behavior for each seismic section, where it is indicated zones of low negative pressure contrast with respect to the layer interpreted as the reservoir.

Figure 10 shows the deviatoric pressure between vertical and horizontal, $P_{T}=\frac{1}{2}\left(\sigma_{z z}-\sigma_{x x}\right)$, where is presented a behavior similar to $P_{x}$ for the reservoir and, in addition, intermediate and high pressures in the basement.

Figure 11 shows the solid hydrostatic pressure, $P_{H}=$ $\frac{1}{2}\left(\sigma_{x x}+\sigma_{z z}\right)$, where the low pressure zone (central blue stripe) is located between two high pressure zones (weaker above and stronger below).

Figure 4: Velocity $V_{\mathrm{P}}$. Line L214-0266.

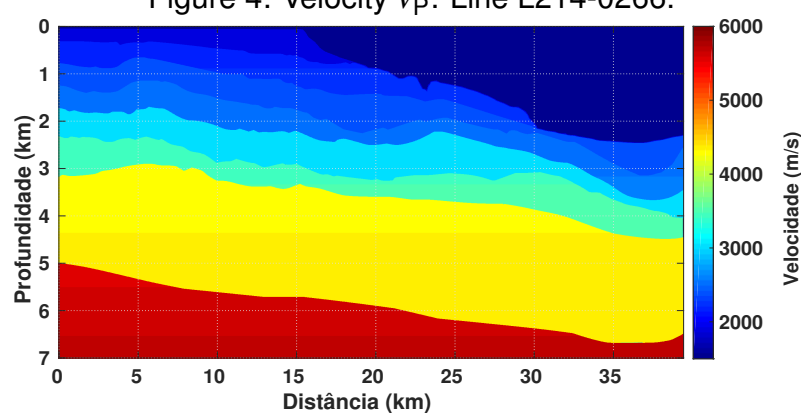

Figure 5: Gamma Ratio $\gamma$. Line L214-0266.

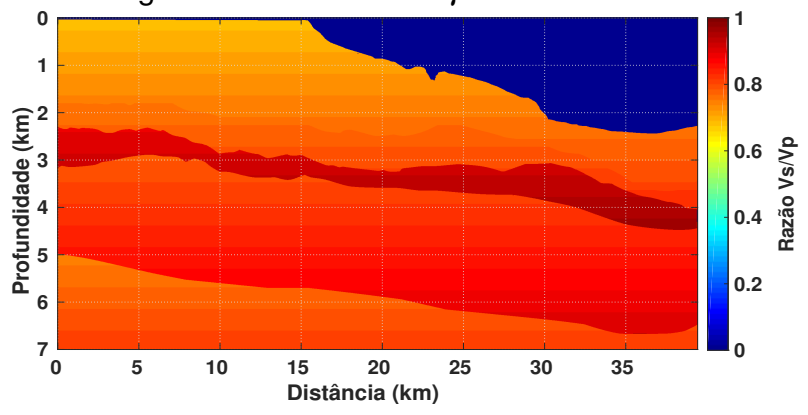

Figure 6: Velocity $V_{\mathrm{S}}$. Line L214-0266.

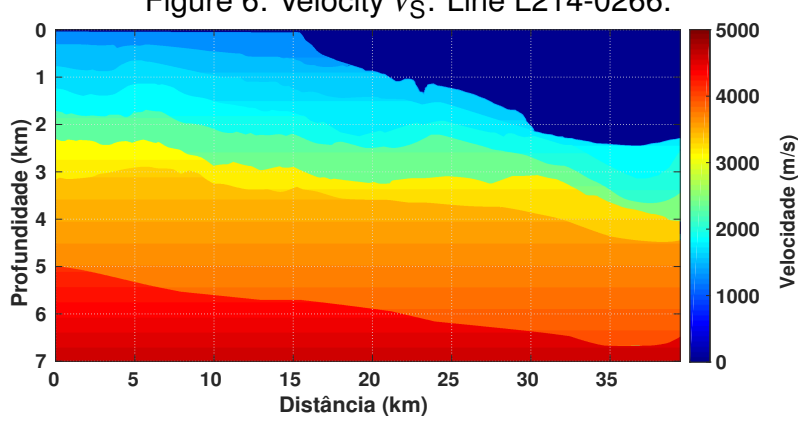

Figure 7: Density $\rho$. Line L214-0266.

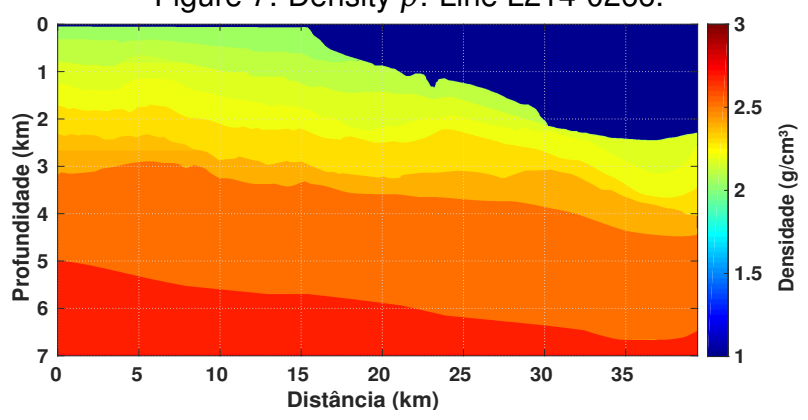

Figure 8: Vertical pressure $P_{z}$. Line L214-0266.

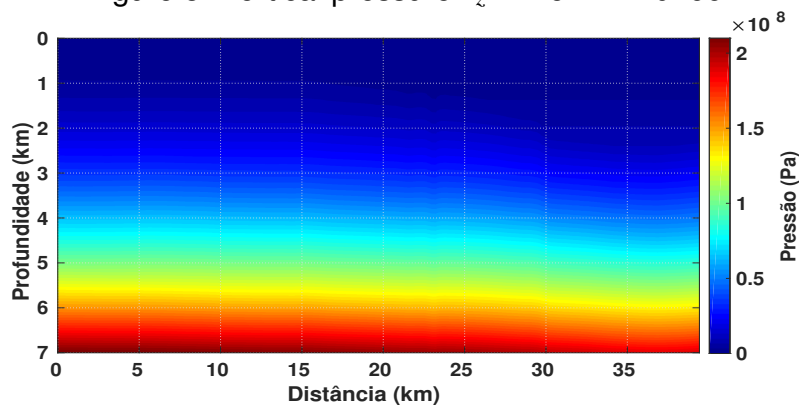


Figure 9: Horizontal pressure $P_{x}$. Line L214-0266.

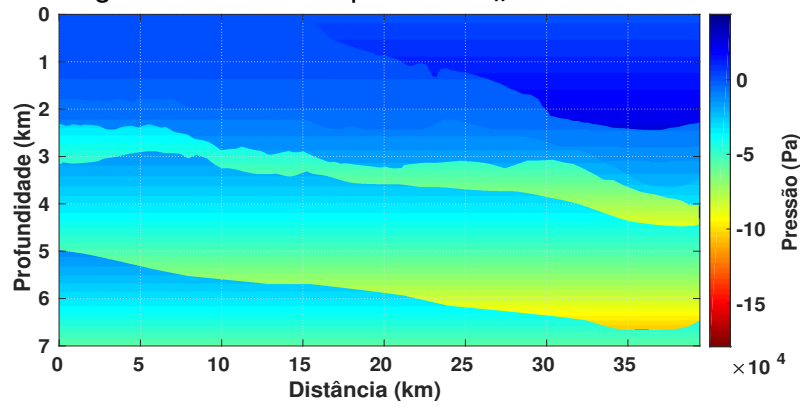

Figure 10: Tangential pressure $P_{T}$. Line L214-0266.

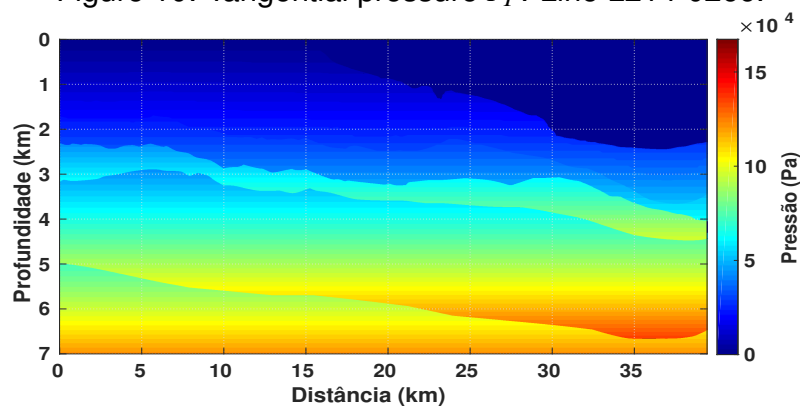

Figure 11: Hydrostatic pressure $P_{H}$. Line L214-0266.

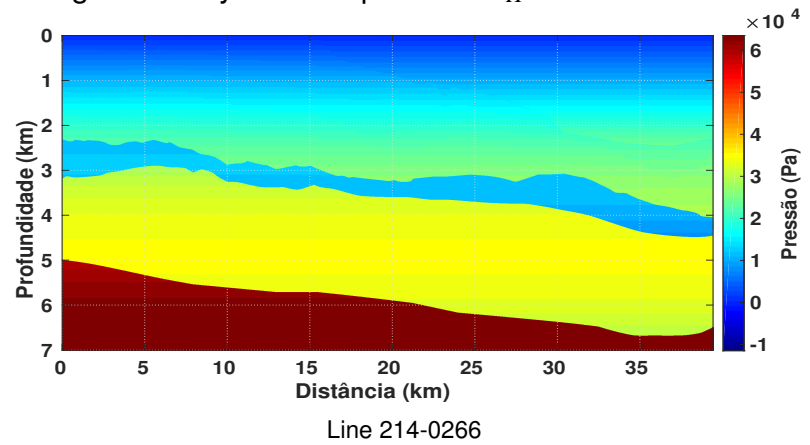

\section{Analysis of the data cubes for the block area}

The next three figures show the obtained interpolated cubes from the correspondent parameters for the the six seismic lines. This analysis is the most important, because the geology makes sense only three-dimensionally.

Figure 12 shows the $3 \mathrm{D}$ cube for the horizontal pressure, where we can follow, with more evidence, the spatial distribution of this zone across part of the cube.

Figure 13 shows the 3D cube constructed for the tangential pressure, where the highest deformation that can take place are located along the orange-red zones.

Figure 14 shows the 3D cube constructed for the solid hydrostatic pressure. This cube clearly separates the zone interpreted as reservoir (central dark blue band in the middle of the cube), that corresponds to the lowest pressure zone. This image should help to extend threedimensionally the zone containing the possible reservoir.
Figure 12: Cube for horizontal pressure $P_{x}$.

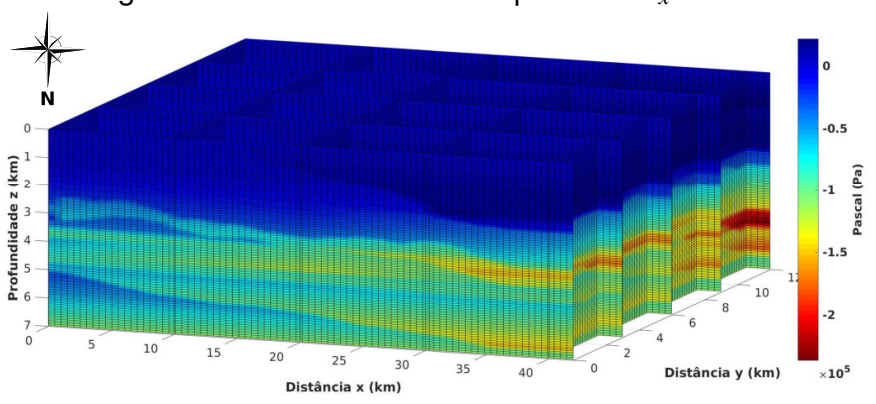

Figure 13: Cube for tangential pressure $P_{T}$.

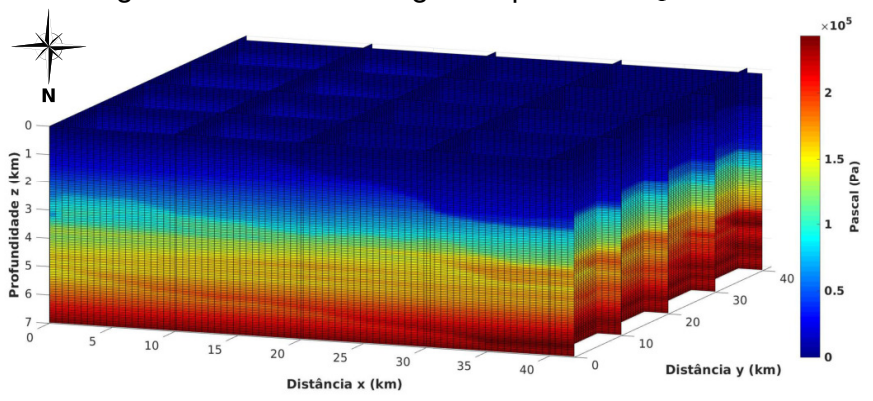

Figure 14: Cube for hydrostatic pressure $P_{H}$.

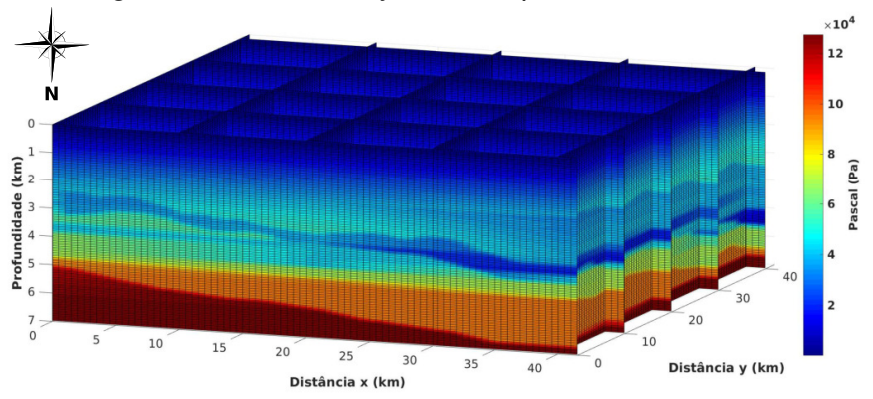

Figures 15 and 16 show horizontal sections extracted from the cube for hydrostatic pressure (figure 14), along the depth range including the zone of lower pressure (approximately $3400 \mathrm{~m}$ to $4800 \mathrm{~m}$ ). These figures presenting the evolution, in depth, of the possible reservoir, displaying the three-dimensional distribution of the attractive fluid zones, represented by the purple areas.

\section{CONCLUSIONS}

The seismic data were processed with CRS technology, which proved to be more effective in the geometrical delineation of reflectors using the stacked and migrated sections to construct an empirical velocity and density models.

The work demonstrated to be possible to estimate and map low pressure zones using velocity maps $V_{P}, V_{S}$ and density maps $\rho$ as starting point. 
Figure 15: Horizontal sections extracted from the cube for hydrostatic pressure. Depth varies between $3400 \mathrm{~m}$ and $4000 \mathrm{~m}$, with increment of $200 \mathrm{~m}$.
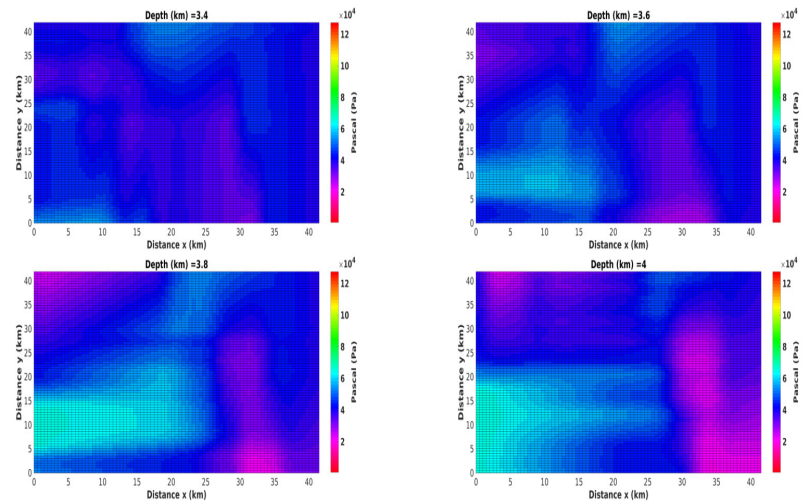

Figure 16: Horizontal sections extracted from the cube for hydrostatic pressure. Depth varies between $4200 \mathrm{~m}$ and $4800 \mathrm{~m}$, with increment of $200 \mathrm{~m}$.
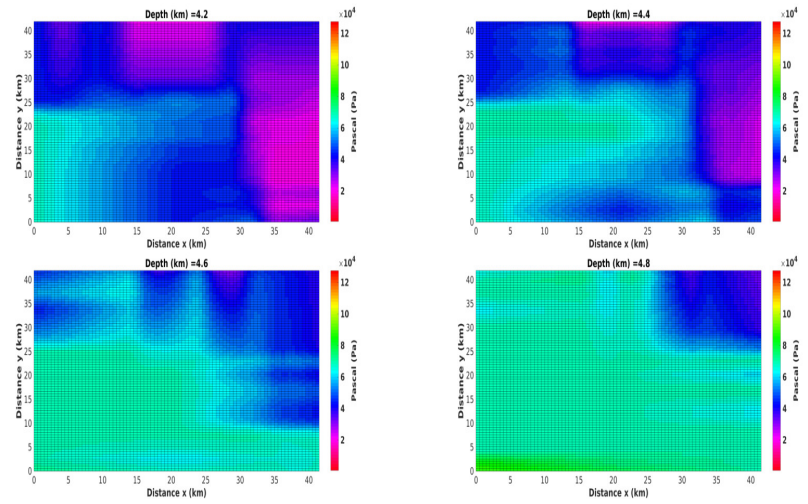

The imaging of low pressure zones, corresponding to a proposed reservoir rock, has a spatial extension that depends on the seismo-stratigraphic and structural interpretation of the sedimentary basin.

The hydrostatic pressure in the solids is defined as average of normal pressure (tensions) $\left(\sigma_{i i}, i=1,2,3\right)$, and constitutes an invariant in the stress-strain state of the solids. Thus, the concept of sealing cap rock does not need to be associated only to petrographic concepts, but also to the concept of a high pressure formation on the top a low pressure formation.

A deeper continuation of this work requires borehole, seismic, geochemical, and geology petroleum information for the area to compose a more complete 3D model.

\section{References}

Andrade, F. T. B., 2018, Modelo 3d da predição de pressão para um bloco da bacia do jequitinhonha: Master's thesis, UFPA, Belém, Pará, Brazil.

Cohen, J. K., and Stockwell, J. J. W., 2005, CWP/SU: Seismic $U n^{*} x$ release 39 - a free package for seismic research and processing: Center for Wave Phenomena, Colorado School of Mines.

Gardner, G. H. F., Gardner, L. W., and Gregory, A. R., 1974, Formation velocity and density - the diagnostic basics for stratigraphic traps: Geophysics, 39, 770-780.

Gregory, A. R., 1976, Fluid saturation effect on dynamic elastic properties of sedimentary rocks: Geophysics, 41, no. 5, 895-921.

Mann, J., 2002, Extensions and aplications of the common reflection surface stack method: Ph.D. thesis, Karlsruhe University, Karlsruhe.

Payton, C. E., 1977, (editor). seismic stratigraphy applications to hydrocarbon exploration: American Association of Petroleum Geologists. Memoir 26, Tulsa, OK.

Persen, L. N., 1975, Rock dynamics and geophysical exploration: Elsevier Scientific Publishing Company, Amsterdam, Holland.

Sibiryakov, E. P., Leite, L. W. B., and Vieira, W. W. S., 2014, Model of the structured continuum, and the relation between specific surface area, porosity and permeability: Brazilian Journal of Geophysics, 31, no. 4, 559-568.

Sibiryakov, E. P., Leite, L. W. B., and Vieira, W. W. S., 2015, Behavior of stresses in structures and the effect on hydrodynamics analyzed from multicomponent seismic data: Brazilian Journal of Geophysics, 33, no. 1, 57-70.

\section{ACKNOWLEDGMENTS}

The authors would like to thank CNPq for the scholarship, and to the Project INCT-GP that gives support to this research aiming at oil exploration. 